\title{
Low-Voltage X-Ray Microanalysis with an Annular Si-Drift
}

\section{Detector}

Paul G. Kotula and Michael T. Brumbach

Sandia National Laboratories, PO Box 5800, Albuquerque, NM 87185-0886

\begin{abstract}
Efforts to improve the spatial resolution of X-ray microanalysis in the SEM have focused on two strategies: Using low primary beam energies to decrease the interaction volume for generation of x-rays [1,2], and using a thin sample and higher primary beam energies (e.g., 20-30 keV, so called STEM-in-SEM) [1,3]. Both methods have advantages and disadvantages. Low-voltage microanalysis while typically well suited to bulk polished surfaces, has a distinct disadvantage in that the lower in primary beam energy we go, the fewer x-ray lines we have available and the more likely that those we do detect will be pathologically overlapped with lines from other elements. A good example of the lowvoltage SEM approach is in the 3D x-ray microanalysis (tomographic spectral imaging) of materials via serial sectioning in the FIB-SEM [3]. In this case making separate thin sections is impractical and therefore low-voltage microanalysis coupled with multivariate statistical analysis to separate the peak overlaps was the best path forward $[2,4]$. The problem of analyzing rough surfaces can be overcome with a high take-off-angle annular Si-drift X-ray detector as used previously for STEM in SEM [3]. In this work, an example of low-voltage X-ray microanalysis in the SEM will be presented where we have used our annular 1 steradian solid-angle Si-drift detector [5] to analyze smooth and rough surfaces at $5 \mathrm{keV}$ or below.
\end{abstract}

A $5 \mathrm{keV}$ primary-beam-energy SEM x-ray spectral image was acquired with a Zeiss Supra 55VP equipped with a Bruker AXS Model 5060 annular geometry Si-drift detector [5] from a Nb-O/Ru-O thin film with significant roughness. The X-ray spectrometer was fitted with a 1 micron Mylar film which allowed good sensitivity to soft X-rays such as the carbon- and oxygen-K lines. An x-ray spectral image consisting of 512 by 384 pixels each with 512 channels was acquired at a microscope magnification of $15 \mathrm{kX}$ resulting in a pixel size of $\sim 14 \mathrm{~nm}$ in 20 seconds with a per pixel total dwell time of 80 microseconds. The average count-rate from the specimen was $120 \mathrm{kcps}$ at a deadtime of $10 \%$ which results in approximately 10 counts per spectrum in the spectral image. Although this may seem like too few counts to work with, the MSA techniques effectively exploit such noisy data. Figure 1 shows the results of the multivariate statistical analysis (MSA) via the methods described in [2,4]. Figure 2 shows an intensity profile across the Ru-rich crystallite marked " 1 " in Fig. 1 . While higher pixel density is needed to better define the microanalytical resolution, it is nonetheless clear that in this situation it is better than 50 nm.

[1] J.I. Goldstein et al., Scanning Electron Microscopy and X-Ray Microanalysis, $3^{\text {rd }}$ Ed. Klewer Academic/Plenum Publishers, New York 2003. 
[2] P.G. Kotula, M.R. Keenan and J.R. Michael. Microsc. Microanal. (2006) 12 36-48.

[3] P.G. Kotula, Microsc. Microanal. (2009) 15 Suppl. 2, 474CD

[4] P.G. Kotula, M.R. Keenan and J.R. Michael. Microsc. Microanal. (2003) 9 1-17.

[5] P.G. Kotula et al., Microsc. Microanal. (2008) 14 116CD.

The author would like to thank Bonnie McKenzie for spectral image acquisition. Sandia is a multiprogram laboratory operated by Sandia Corporation, a Lockheed Martin Company, for the United Stated Department of Energy's (DOE) National Nuclear Security Administration (NNSA) under contract DE-AC0494AL85000.
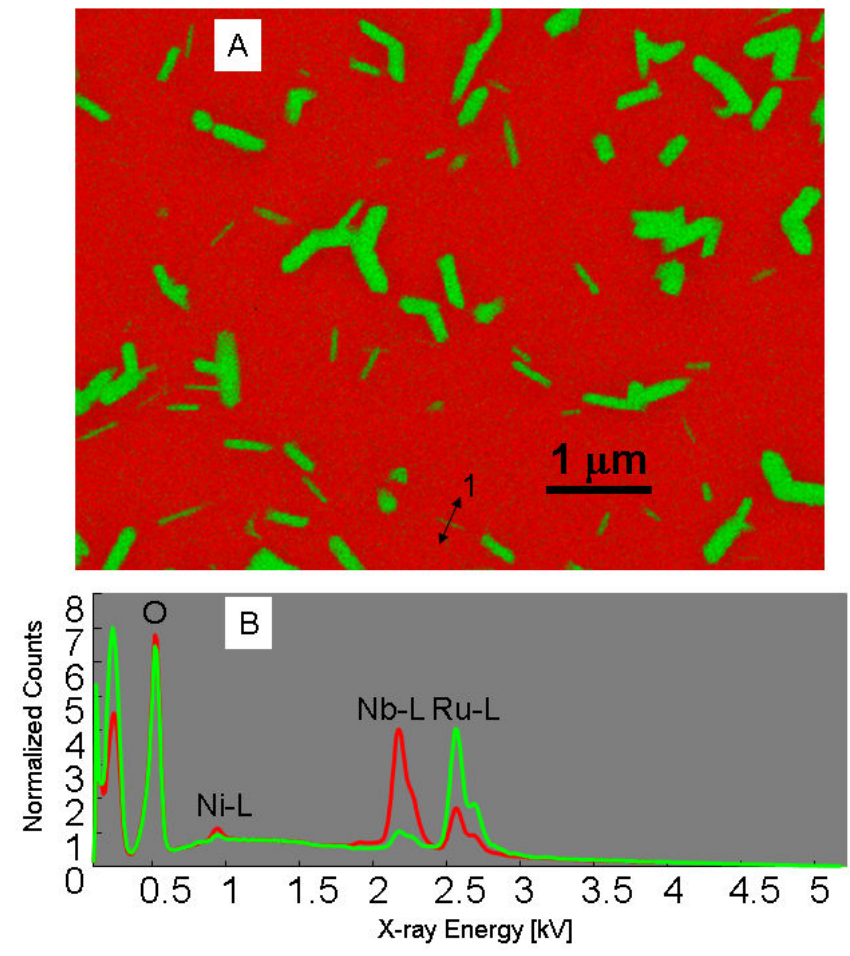

Figure 1. MSA results from $5 \mathrm{kV}$ SEM with annular Si-drift detector EDS, spectral image. Red is a Nb-rich oxide film and green are Ru-rich oxide crystallites.
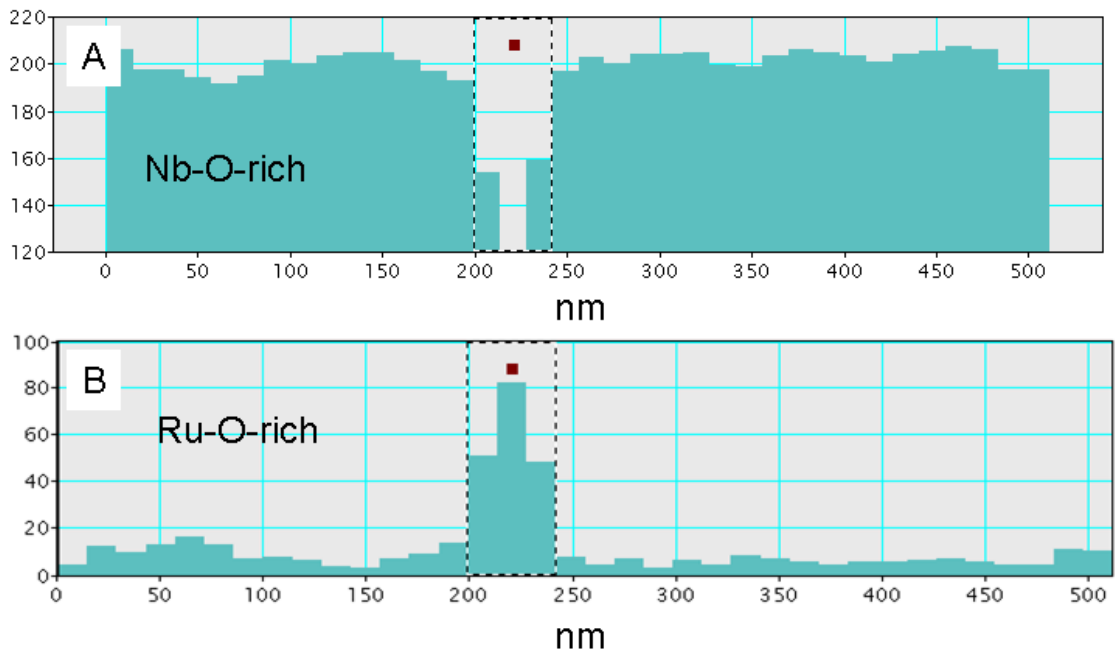

Figure 2. Component image intensity profiles extracted from the arrow marked " 1 " in Fig. 1. 